\title{
Aspectos ecológicos das espécies regenerantes de uma floresta urbana com 150 anos de sucessão florestal: 0 risco das espécies exóticas
}

\author{
Ecological aspects of regenerating species of an urban forest with 150 years old of forest \\ succession: the risk of exotic species
}

\author{
Lucas Deziderio Santana ${ }^{\mathrm{I}}$, Cassiano Ribeiro da Fonseca ${ }^{\mathrm{II}}$, Fabrício Alvim Carvalho ${ }^{\mathrm{III}}$
}

\begin{abstract}
Resumo
A presença de florestas dentro de centros urbanos tem grande importância, principalmente devido aos serviços ecossistêmicos proporcionados por elas. Fragmentos urbanos são pouco estudados e trabalhos quantitativos focados na flora arbórea na área do presente estudo nunca foram realizados. O objetivo foi avaliar a composição, estrutura e diversidade de espécies regenerantes de uma floresta urbana, oriunda de um projeto paisagístico. Buscou-se responder a seguinte questão: a floresta está conseguindo avançar no processo sucessional após 150 anos de regeneração natural? O estudo foi realizado em uma área com tamanho total de aproximadamente 7,8 ha e de predomínio da Floresta Atlântica. A amostragem foi realizada em 25 parcelas de $5 \times 5 \mathrm{~m}$, nas quais todos os indivíduos com diâmetro a altura do peito (DAP) $<5 \mathrm{~cm}$ e altura $>1 \mathrm{~m}$ foram amostrados. Encontram-se 1297 indivíduos, distribuídos em 84 espécies. Três espécies exóticas com conhecido potencial invasor foram encontradas: Coffea arabica, Artocarpus heterophyllus e Persea americana. A espécie Coffea arabica apresentou o maior Valor de Importância na comunidade. $\mathrm{O}$ índice de diversidade de Shannon foi de $\mathrm{H}^{\prime}=3,10$ nats.ind ${ }^{-1}$, e equabilidade de Pielou de $\mathrm{J}^{\prime}=0,70$. A análise de Cluster (coeficiente de Morisita-Horn) evidenciou uma comunidade com baixa heterogeneidade interna. A análise dos grupos ecológicos revelou uma comunidade com dificuldades em avançar para estágios sucessionais superiores. Este estudo é importante para reforçar o risco das espécies exóticas invasoras em projetos paisagísticos.
\end{abstract}

Palavras-chave: Coffea arabica; Espécies exóticas; Fitossociologia; Regeneração

\begin{abstract}
The presence of forest within urban centers has great importance, especially in relation to ecosystem services provided by them. Urban forests are poorly studied. Moreover, quantitative studies focused on tree flora of the present study area remain to be established. Here, we evaluated the composition, structure and diversity of species in regeneration from an urban forest, originating from a landscaping design. We sought to answer the following question: Did the forest advance in the succession process after 150 years of regeneration? The survey was done on 25 plots of $5 \times 5$ $\mathrm{m}$, where all the individuals with diameter at breast height $(\mathrm{DBH})<5 \mathrm{~cm}$ and height $>1 \mathrm{~m}$ were sampled. We found 1297 individuals, distributed in 84 species. Three alien species with potential invasive acquaintance were found: Coffea arabica, Artocarpus heterophyllus and Persea americana. Coffea arabica showed the highest importance value in the community. Shannon diversity index was $H^{\prime}=3,10$ nats.ind ${ }^{-1}$, and Pielou equability $\mathrm{J}^{\prime}=0.70$. The cluster analysis (Morisita-Horn coefficient) showed a community with low internal heterogeneity. The analysis of ecological groups revealed a community with predominantly early successional groups. Despite of 150 years of natural regeneration, our findings indicated a community with difficulties in advancing to higher successional stages. This study is important to highlight the risk of invasive alien species in landscaping designs.
\end{abstract}

Keywords: Coffea arabica; Alien species; Phytosociology; Regeneration

\footnotetext{
I Biólogo, MSc., Doutorando em Engenharia Florestal, Laboratório de Ecologia Vegetal, Universidade Federal de Lavras, Avenida Doutor Sylvio Menicucci, 1001, Kennedy, CEP 37200-000, Lavras (MG), Brasil. lucasdeziderio01@gmail.com (ORCID: 0000-0003-1582-7513)

II Biólogo, Dr., Professor do Instituto Federal de Educação, Ciência e Tecnologia do Sudeste de Minas Gerais, CEP 36080-001, Juiz de Fora (MG), Brasil. cassianoribeirofonseca@gmail.com (ORCID: 0000-0002-9230-3185)

III Biólogo, Dr., Professor do Departamento de Botânica, Universidade Federal de Juiz de Fora, Rua José Lourenço Kelmer, s/n, Martelos, CEP 36036-330, Juiz de Fora (MG), Brasil. fabricio.alvim@gmail.com (ORCID: 0000-0001-7301-9448)
} 


\section{Introdução}

Em função da fragmentação e destruição dos ecossistemas naturais, a presença de vegetação dentro dos centros urbanos vem adquirindo extrema importância (ALVEY, 2006; PERRING et al., 2013), sobretudo com relação aos serviços ecossistêmicos que eles proporcionam. Perring et al. (2013) comentam que florestas urbanas proporcionam melhor qualidade do ar local, mantêm a biodiversidade, melhoram o bem-estar humano, através da regulação do clima, redução do ruído, recreação e educação ao ar livre e ainda têm o potencial de sequestrar e armazenar carbono da atmosfera. Além disso, informações sobre os padrões ecológicos de uma floresta urbana podem ser usados como parâmetros para subsidiar ações de recuperação e restauração ecológica em nível local (ALVEY, 2006).

No entanto, as florestas urbanas estão muito vulneráveis a ações antrópicas, com isso, tendem a ser mais perturbadas e principalmente mais suscetíveis às invasões biológicas (PERRING et al., 2013). A presença frequente de espécies exóticas em área urbana funciona como um importante centro irradiador de invasões biológicas e pode ser considerada uma das principais causas de homogeneização biológica em escala global (MCKINNEY, 2006). A inserção de espécies exóticas em projetos paisagísticos no Brasil sempre foi comum (GONÇALVES; PAIVA, 2004), porém, muitas espécies exóticas podem se tornar invasoras. As espécies exóticas invasoras são consideradas a segunda maior causa de extinção de espécies no planeta, afetando diretamente a biodiversidade, a economia e a saúde humana (PIMENTEL et al., 2001). Espécies exóticas invasoras também causam impactos aos ecossistemas, uma vez que modificam os ciclos ecológicos naturais, afetando os serviços por eles prestados (MCKINNEY, 2006).

$\mathrm{O}$ risco das espécies exóticas nem sempre foram levados em consideração, como pode ser constatado através dos primeiros projetos de recuperação florestal no Brasil. Estes projetos foram iniciados principalmente na segunda metade do século XIX, na região da Tijuca (Rio de Janeiro) (BANDEIRA, 1993; ABREU; RODRIGUES, 2010; DURIGAN; ENGEL, 2012), período este que também foi realizado a recuperação florestal na área do presente estudo. Contudo, as iniciativas de recuperação florestal não tinham um conhecimento técnico-científico formado, apesar de muitos terem sido bem-sucedidos (DURIGAN; ENGEL, 2012). Desta forma, a inserção de espécies exóticas nos projetos não era vista como algo prejudicial à biodiversidade. Entretanto, o que se percebe hoje em muito destes lugares é a dominância destas espécies tanto em densidade quanto em biomassa (ABREU; RODRIGUES, 2010). Nas últimas décadas, estudos que buscam recuperar ecossistemas no Brasil têm crescido bastante, com isso, áreas que antes eram vistas como degradadas, atualmente, com o auxílio das técnicas de recuperação florestal, estão regenerando (RODRIGUES et al., 2009; DURIGAN; ENGEL, 2012; MELI et al., 2017).

A regeneração é um processo variável no tempo e no espaço, refere-se às fases iniciais do ciclo de progresso da floresta e representa uma fase importante para a sobrevivência, crescimento e manutenção da comunidade (CHAZDON, 2008). Com isso, os estudos de regeneração propiciam a realização de previsões sobre o comportamento e o desenvolvimento futuro da floresta, além de fornecer a relação e a quantidade de espécies que constituem o seu estoque, bem como suas dimensões e distribuição (CHAZDON, 2008).

Em sua maioria, os fragmentos florestais na Zona da Mata Mineira, Sudeste do Brasil, são oriundos do abandono de áreas que no passado serviam para a produção agrícola cafeeira ou como pastagem (VALVERDE, 1958; DRUMMOND et al., 2005). Atualmente, estas florestas encontram-se em fragmentos isolados na paisagem e em diferentes estágios sucessionais. Situação bem diferente das condições originais, que formavam densas florestas e cobria toda região, fato que deu origem ao nome Zona da Mata (VALVERDE, 1958; MEIRA-NETO et al., 1997). Além disso, a urbanização também contribui bastante para o desmatamento de grandes áreas e para o isolamento destas florestas (DRUMMOND et al., 2005).

Levando em consideração a importância das florestas urbanas para a manutenção da biodiversidade e para o bem-estar humano (PERRING et al., 2013) e sabendo que estudos quantitativos da vegetação arbórea da área do presente estudo nunca foram realizados, o objetivo do trabalho foi avaliar a composição, estrutura e diversidade de espécies arbustivas-arbóreas do estrato regenerante de uma floresta urbana, oriundo de um projeto paisagístico e com uma expressiva presença de espécies exóticas, assim como inferir as tendências futuras da comunidade. Para tanto, buscou-se responder a seguinte pergunta: a floresta urbana está avançando no processo sucessional após 150 anos de regeneração? 


\section{Material e métodos}

Área de estudo

O estudo foi realizado no Parque Mariano Procópio (MAPRO) (214'45.56” S, 43²1’34.66” O), Juiz de Fora, Minas Gerais, Brasil (Figura 1-A). O município está situado na mesorregião da Zona da Mata Mineira, com altitudes que variam em torno de $800 \mathrm{~m}$ acima do nível do mar (JUIZ DE FORA, 2016). O MAPRO é oriundo de um projeto paisagístico datado de 1861, atribuído a Auguste François Marie Glaziou. Antes da intervenção do paisagista, o local era uma área desmatada para a plantação de café (DELPHIM, 2007) (Figura 1-B). Atualmente, o MAPRO abrange uma área de $78.240 \mathrm{~m}^{2}$ (7,8 ha), em processo de sucessão secundária há cerca de 150 anos, localizado em plena área urbana de Juiz de Fora (Figura 1-C, D). Apesar de ser um pequeno fragmento florestal (7,8 ha), o MAPRO está situado entre três grandes remanescentes florestais na zona urbana do município de Juiz de Fora: a 1,2 km do Morro do Imperador (floresta de 78 ha), a 1,5 km da Mata do Krambeck (370 ha), e a 3,5 km da Reserva Biológica Municipal Poço D'Anta (277 ha).

Figura 1 - (A) Localização geográfica do Parque Mariano Procópio (Juiz de Fora - MG, Brasil). (B) Início do projeto de implementação da vegetação do Parque em 1861. Foto: Revert Henry Klumb. (C) Vista aérea do Parque Mariano Procópio em 1939; Arquivo: Ramon Brandão. (D) Vista aérea do Parque em 2014.

Figure 1 - (A) Geographical location of Park 'Mariano Procópio' (Juiz de Fora - MG state, Brazil). (B) The start of the implementation project for the vegetation of the park in 1861. Photograph: Revert Henry Klumb. (C)

Aerial view of Park 'Mariano Procópio' in 1939; File: Ramon Brandão. (D) Aerial view of the Park in 2014.

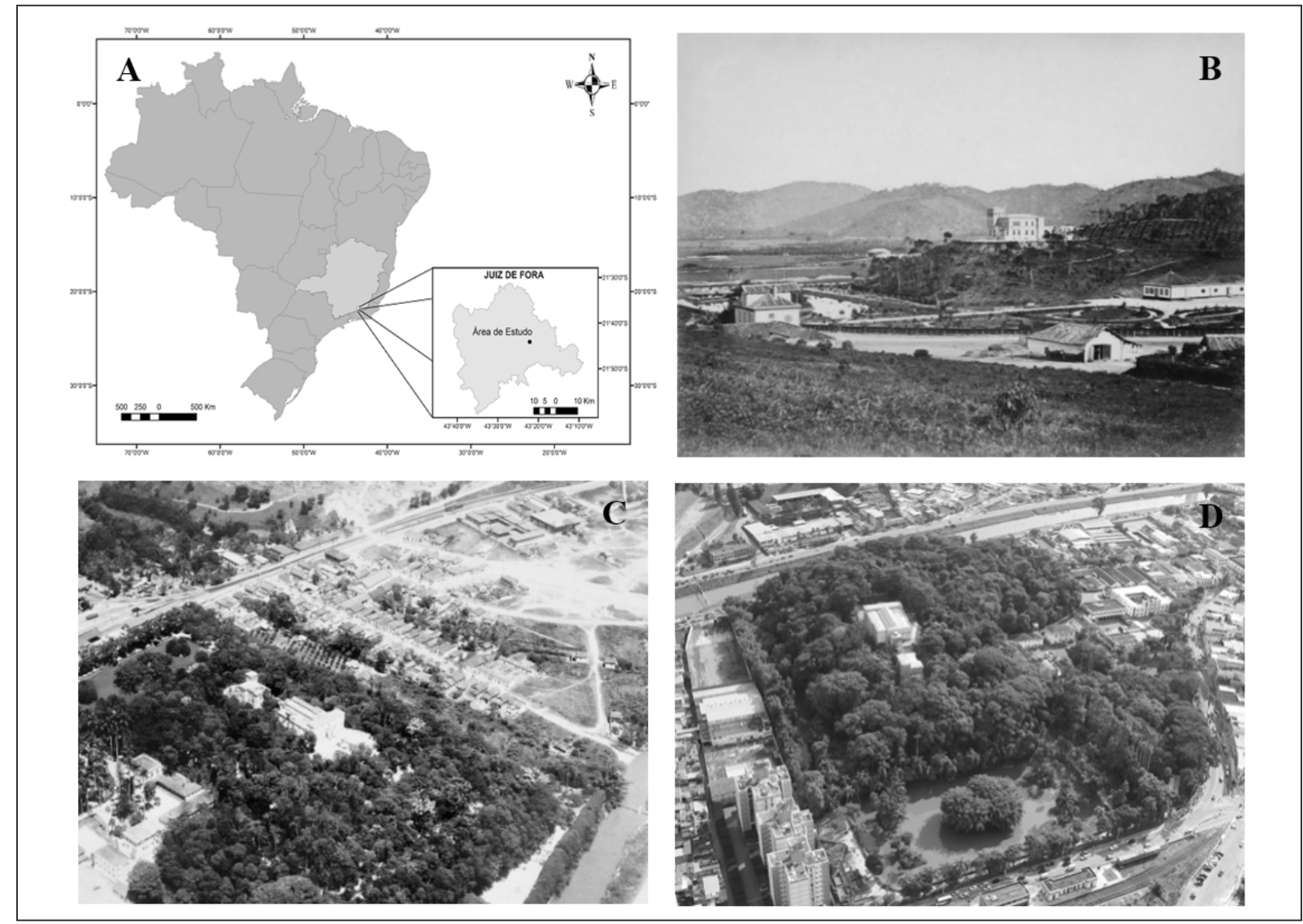

Fonte: http://mapro.pjf.mg.gov.br/index.php/apresentacao; http://www.mariadoresguardo.com.br/2010/01/museu-mariano-procopio-arquivo-ramon. html e http://www.tribunademinas.com.br/cultura/o-verde-em-meio-a-tanto-cinza-1.1451287.

Source: http://mapro.pjf.mg.gov.br/index.php/apresentacao; http://www.mariadoresguardo.com.br/2010/01/museu-mariano-procopio-arquivo-ramon. html and http://www.tribunademinas.com.br/cultura/o-verde-em-meio-a-tanto-cinza-1.1451287. 
Na região, a fitofisionomia predominante é da Floresta Estacional Semidecidual Montana (IBGE, 2012). O relevo é formado por rochas antigas, basicamente gnaisses e granitos e com solo predominante do tipo Latossolo Vermelho Amarelo Distrófico. Segundo a classificação de Köppen, a região possui clima Cwa, caracterizando-se por possuir duas estações bem definidas, verão - primavera com temperaturas mais elevadas e maior precipitação pluviométrica (outubro a abril) e outono - inverno mais frio e com menor precipitação (maio a setembro). A pluviosidade média anual fica próxima a $1.500 \mathrm{~mm}$, com taxas mais altas em Janeiro $(\approx 300 \mathrm{~mm})$. A temperatura média anual oscila em torno de $18,9^{\circ} \mathrm{C}$, o mês mais quente (fevereiro) possui média próxima a $21,7^{\circ} \mathrm{C}$ e o mês mais frio (julho) $16,1^{\circ} \mathrm{C}$ (JUIZ DE FORA, 2016)

\section{Amostragem e coleta dos dados}

Para a alocação das parcelas foram excluídas regiões que eram ocupadas pelos pomares, principalmente regiões que eram exclusivas das jabuticabeiras. As demais áreas foram consideradas como universo amostral, nas quais foram alocadas aleatoriamente 25 parcelas de 5 x $5 \mathrm{~m}$. Todos os indivíduos arbóreos vivos com altura maior que $1 \mathrm{~m}$ e DAP $<5 \mathrm{~cm}$ (DAP = diâmetro a altura do peito a $1,30 \mathrm{~cm}$ acima do nível do solo) foram amostrados e mensurados quanto ao DAS (DAS = diâmetro a altura do solo a $30 \mathrm{~cm}$ do solo) e identificação botânica. A coleta dos dados foi realizada durante os meses de maio a agosto de 2013.

A sinonímia e a grafia das espécies foram checadas segundo a Lista da Flora do Brasil 2020, seguindo a classificação das famílias botânicas, conforme a proposta do Angiosperm Phylogeny Group IV (ANGIOSPERM PHYLOGENY GROUP, 2016). O material botânico coletado foi identificado por comparação utilizando a coleção do Laboratório de Ecologia Vegetal da Universidade Federal de Juiz de Fora (UFJF) e do herbário (CESJ) da UFJF, além de consultas a especialistas.

Análise dos dados

Os parâmetros fitossociológicos calculados através do software Microsoft Office Excel $2010^{\circledR}$ foram analisados segundo Kent e Coker (1992), obtendo-se: área basal (AB), frequência absoluta (FA), frequência relativa (FR), densidade absoluta (DA), densidade relativa (DR), dominância relativa (DoR) e Valor de Importância (VI).

A diversidade de espécies foi analisada através do índice de diversidade de Shannon (H'). O índice de equabilidade de Pielou (J), baseado em H', foi utilizado para estimativa da uniformidade da comunidade (MAGURRAN, 2004). Para explorar a formação de grupos de parcelas e espécies, uma análise utilizando o método de agrupamento UPGMA foi aplicada a partir do coeficiente quantitativo de Morisita-Horn (FELFILI et al., 2011). De forma complementar, para verificar a formação de gradientes ambientais na comunidade, uma Análise de Correspondência Distendida (DCA) foi utilizada. Para a realização desta análise foi elaborada uma matriz de densidade de espécies por parcelas, sendo incluídas apenas as espécies com mais de 10 indivíduos, segundo proposto por Felfili et al. (2011). As análises foram realizadas no software PAST 3.10 (HAMMER et al., 2001).

A distribuição geográfica das espécies identificadas foi obtida através de consulta à Lista de Espécies da Flora do Brasil 2020 e foram definidos seis padrões de distribuição das espécies: 1) exclusivas da Floresta Atlântica, 2) ocorrentes no Cerrado e Floresta Atlântica, 3) ocorrentes na Caatinga e Floresta Atlântica e 4) ocorrentes em três ou mais domínios fitogeográficos (ampla distribuição), 5) nativas do Brasil, porém, não possui distribuição geográfica natural na floresta estacional semidecidual e 6) exóticas e exóticas invasoras de fora do Brasil. Para classificação das espécies exóticas invasoras no Brasil foi utilizado o banco de dados nacional de espécies exóticas invasoras, I3N Brasil, do Instituto Hórus de Desenvolvimento e Conservação Ambiental (INTER-AMERICAN BIODIVERSITY INFORMATION NETWORK, 2014).

A classificação das espécies em grupos sucessionais seguiu o modelo proposto por Oliveira-Filho e Scolforo (2008), considerando-se as características ecológicas e sucessionais das espécies para classificálas, tendo como principal fator de inclusão nas categorias, a quantidade de luz de que dependem para seu desenvolvimento, sendo: Pioneiras (Pi), secundárias iniciais (SI) e secundárias tardias (ST). Por estarem em uma área fora do seu país de origem, com isso, podendo apresentar diferente estratégia de crescimento, as espécies exóticas não foram classificadas, criando a categoria Exot (Exótica). 


\section{Resultados e discussão}

$\mathrm{Na}$ comunidade regenerante foram amostrados 1297 indivíduos (20.752 ind.ha $\left.{ }^{-1}\right)$, pertencentes a 30 famílias, 57 gêneros e 84 espécies, sendo que quatro espécies foram identificadas até o nível de família, oito até o nível de gênero e duas como indeterminadas (Tabela 1).

Tabela 1 - Parâmetros fitossociológicos das espécies regenerantes do fragmento florestal do Parque Mariano Procópio, Juiz de Fora - Minas Gerais. Espécies ordenadas de forma decrescente segundo o valor de importância (VI \%).

Table 1 - Phytosociological parameters of species in regeneration of the forest fragment of the Park 'Mariano Procópio', Juiz de Fora - Minas Gerais state. Ordered species in decreasing order according to importance value (VI\%).

\begin{tabular}{|c|c|c|c|c|c|c|c|c|}
\hline Espécie & Família & GS & DG & DA & DR & DoR & FR & VI (\%) \\
\hline Coffea arabica L.* & Rubiaceae & $\mathrm{NC}$ & Exot & 284 & 21,9 & 16,91 & 5,97 & 14,93 \\
\hline Nectandra nitidula Nees & Lauraceae & $\mathrm{Pi}$ & Amp & 135 & 10,41 & 12,84 & 5,97 & 9,74 \\
\hline $\begin{array}{l}\text { Myrciaria glazioviana } \\
\text { (Kiaersk.).M.Barroso ex Sobral }\end{array}$ & Myrtaceae & SI & S/FES & 118 & 9,1 & 9,96 & 4,09 & 7,72 \\
\hline Cupania oblongifolia Mart. & Sapindaceae & $\mathrm{Pi}$ & Amp & 89 & 6,86 & 9 & 6,6 & 7,49 \\
\hline Erythroxylum citrifolium A.St.-Hil. & Erythroxylaceae & SI & Amp & 73 & 5,63 & 8,2 & 6,6 & 6,81 \\
\hline $\begin{array}{l}\text { Pseudopiptadenia contorta (DC.) } \\
\text { G.P.Lewis \& M.P.Lima }\end{array}$ & Fabaceae & SI & Amp & 111 & 8,56 & 3,2 & 2,83 & 4,86 \\
\hline Artocarpus heterophyllus Lam.* & Moraceae & $\mathrm{NC}$ & Exot & 54 & 4,16 & 5,31 & 2,83 & 4,1 \\
\hline Myrcia splendens (Sw.) DC. & Myrtaceae & $\mathrm{Pi}$ & Amp & 23 & 1,77 & 0,9 & 4,09 & 2,25 \\
\hline Myrcia multiflora (Lam.) DC. & Myrtaceae & SI & Amp & 30 & 2,31 & 3,13 & 0,94 & 2,13 \\
\hline Plinia cauliflora (Mart.) Kausel & Myrtaceae & $\mathrm{Pi}$ & $\mathrm{S} / \mathrm{FES}$ & 15 & 1,16 & 1,88 & 2,51 & 1,85 \\
\hline Lecythis pisonis Cambess. & Lecythidaceae & ST & $\mathrm{S} / \mathrm{FES}$ & 19 & 1,46 & 2,5 & 1,89 & 1,77 \\
\hline Brosimum guianense (Aubl.) Huber & Moraceae & SI & Amp & 12 & 0,93 & 1,34 & 2,52 & 1,77 \\
\hline Machaerium brasiliense Vogel & Fabaceae & $\mathrm{Pi}$ & Amp & 17 & 1,31 & 1 & 2,52 & 1,61 \\
\hline Faramea multiflora A.Rich. ex DC. & Rubiaceae & SI & $\mathrm{S} / \mathrm{FES}$ & 39 & 3,01 & 0,51 & 0,94 & 1,49 \\
\hline Erythroxylum pelleterianum A.St.-Hil. & Erythroxylaceae & SI & Amp & 16 & 1,23 & 0,62 & 2,2 & 1,35 \\
\hline Miconia latecrenata (DC.) Naudin & Melastomataceae & $\mathrm{Pi}$ & Excl & 14 & 1,08 & 1,48 & 1,26 & 1,27 \\
\hline $\begin{array}{l}\text { Handroanthus chrysotrichus (Mart. ex } \\
\text { DC.) Mattos }\end{array}$ & Bignoniaceae & $\mathrm{Pi}$ & $\mathrm{Ce} / \mathrm{FA}$ & 13 & 1 & 0,92 & 1,89 & 1,27 \\
\hline Guarea guidonia (L.) Sleumer & Meliaceae & ST & Amp & 10 & 0,77 & 0,94 & 1,89 & 1,2 \\
\hline Camellia sinensis (L.) Kuntze * & Theaceae & $\mathrm{NC}$ & Exot & 15 & 1,16 & 0,86 & 1,26 & 1,09 \\
\hline Piper anisum (Spreng.) Angely & Piperaceae & $\mathrm{Pi}$ & $\mathrm{Ce} / \mathrm{FA}$ & 19 & 1,46 & 0,14 & 1,57 & 1,06 \\
\hline Guapira opposita (Vell.) Reitz & Nyctaginaceae & SI & Amp & 8 & 0,62 & 0,97 & 1,57 & 1,05 \\
\hline Inga cylindrica (Vell.) Mart. & Fabaceae & $\mathrm{Pi}$ & Amp & 8 & 0,62 & 0,88 & 1,57 & 1,02 \\
\hline Ilex cerasifolia Reissek & Aquifoliaceae & ST & $\mathrm{Ce} / \mathrm{FA}$ & 8 & 0,62 & 0,2 & 2,2 & 1,01 \\
\hline Cybistax antisyphilitica (Mart.) Mart. & Bignoniaceae & $\mathrm{Pi}$ & Amp & 6 & 0,46 & 0,65 & 1,89 & 1 \\
\hline Xylopia sericea A.St.-Hil. & Annonaceae & $\mathrm{Pi}$ & Amp & 7 & 0,54 & 0,8 & 1,57 & 0,97 \\
\hline Psychotria vellosiana Benth. & Rubiaceae & SI & Amp & 10 & 0,77 & 0,83 & 1,26 & 0,95 \\
\hline Jacaranda micrantha Cham. & Bignoniaceae & SI & Excl & 4 & 0,31 & 1,47 & 0,94 & 0,91 \\
\hline Eugenia florida DC. & Myrtaceae & $\mathrm{Pi}$ & Amp & 8 & 0,62 & 0,47 & 1,57 & 0,89 \\
\hline Bathysa australis (A.St.-Hil.) K.Schum. & Rubiaceae & SI & $\mathrm{Ce} / \mathrm{FA}$ & 5 & 0,39 & 1,15 & 0,63 & 0,72 \\
\hline
\end{tabular}


Tabela 1 - Continuação...

Table 1 - Continuation...

\begin{tabular}{|c|c|c|c|c|c|c|c|c|}
\hline Espécie & Família & GS & DG & DA & DR & DoR & FR & VI (\%) \\
\hline Cupania ludowigii Somner \& Ferrucci & Sapindaceae & $\mathrm{Pi}$ & Excl & 8 & 0,62 & 0,24 & 1,26 & 0,71 \\
\hline $\begin{array}{l}\text { Allophylus edulis (A.St.-Hil. et al.) Hieron. } \\
\text { ex Niederl. }\end{array}$ & Sapindaceae & SI & Amp & 3 & 0,23 & 1,05 & 0,63 & 0,64 \\
\hline Piper tectoniifolium Kunth & Piperaceae & SI & $\mathrm{Ce} / \mathrm{FA}$ & 10 & 0,77 & 0,36 & 0,63 & 0,59 \\
\hline Dalbergia frutescens (Vell.) Britton & Fabaceae & $\mathrm{Pi}$ & Amp & 3 & 0,23 & 0,57 & 0,94 & 0,58 \\
\hline Piper arboreum Aubl. & Piperaceae & SI & Amp & 7 & 0,54 & 0,22 & 0,94 & 0,57 \\
\hline Indeterminada sp1 & Indeterminada & $\mathrm{NC}$ & $\mathrm{NC}$ & 3 & 0,23 & 0,4 & 0,94 & 0,52 \\
\hline Eugenia brasiliensis Lam. & Myrtaceae & SI & Excl & 5 & 0,39 & 0,5 & 0,63 & 0,5 \\
\hline Ocotea laxa (Nees) Mez & Lauraceae & SI & Excl & 3 & 0,23 & 0,19 & 0,94 & 0,46 \\
\hline $\begin{array}{l}\text { Myrsine coriacea (Sw.) R.Br. ex Roem. \& } \\
\text { Schult. }\end{array}$ & Primulaceae & $\mathrm{Pi}$ & $\mathrm{Ce} / \mathrm{FA}$ & 5 & 0,39 & 0,03 & 0,94 & 0,45 \\
\hline Swietenia macrophylla King & Meliaceae & $\mathrm{NC}$ & Amp & 2 & 0,15 & 0,52 & 0,63 & 0,43 \\
\hline Myrcia eriopus DC. & Myrtaceae & SI & $\mathrm{S} / \mathrm{FES}$ & 4 & 0,31 & 0,58 & 0,31 & 0,4 \\
\hline Poincianella pluviosa (DC.) L.P.Queiroz & Fabaceae & $\mathrm{ST}$ & $\mathrm{S} / \mathrm{FES}$ & 4 & 0,31 & 0,25 & 0,63 & 0,39 \\
\hline Pterogyne nitens Tul. & Fabaceae & $\mathrm{Pi}$ & Amp & 3 & 0,23 & 0,22 & 0,63 & 0,36 \\
\hline $\begin{array}{l}\text { Piptadenia gonoacantha (Mart.) } \\
\text { J.F.Macbr. }\end{array}$ & Fabaceae & $\mathrm{Pi}$ & $\mathrm{Ce} / \mathrm{FA}$ & 4 & 0,31 & 0,13 & 0,63 & 0,36 \\
\hline Sorocea guilleminiana Gaudich. & Moraceae & SI & $\mathrm{Ce} / \mathrm{FA}$ & 3 & 0,23 & 0,2 & 0,63 & 0,35 \\
\hline Lonchocarpus sp. & Fabaceae & $\mathrm{NC}$ & $\mathrm{NC}$ & 4 & 0,31 & 0,12 & 0,63 & 0,35 \\
\hline Casearia sylvestris Sw. & Salicaceae & $\mathrm{Pi}$ & Amp & 2 & 0,15 & 0,27 & 0,63 & 0,35 \\
\hline Xylopia brasiliensis Spreng. & Annonaceae & $\mathrm{Pi}$ & Amp & 2 & 0,15 & 0,23 & 0,63 & 0,34 \\
\hline Pterocarpus cf. rohrii Vahl & Fabaceae & $\mathrm{NC}$ & Amp & 2 & 0,15 & 0,2 & 0,63 & 0,33 \\
\hline Machaerium nyctitans (Vell.) Benth. & Fabaceae & $\mathrm{Pi}$ & $\mathrm{Ce} / \mathrm{FA}$ & 3 & 0,23 & 0,08 & 0,63 & 0,31 \\
\hline Siparuna guianensis Aubl. & Siparunaceae & SI & Amp & 3 & 0,23 & 0,07 & 0,63 & 0,31 \\
\hline Myrtaceae sp1 & Myrtaceae & $\mathrm{NC}$ & $\mathrm{NC}$ & 2 & 0,15 & 0,13 & 0,63 & 0,3 \\
\hline Myrcia sp1 & Myrtaceae & $\mathrm{NC}$ & $\mathrm{NC}$ & 1 & 0,08 & 0,49 & 0,31 & 0,29 \\
\hline Myrtaceae sp2 & Myrtaceae & $\mathrm{NC}$ & $\mathrm{NC}$ & 2 & 0,15 & 0,1 & 0,63 & 0,29 \\
\hline Malvaviscus arboreus Cav. * & Malvaceae & $\mathrm{NC}$ & Exot & 4 & 0,31 & 0,22 & 0,31 & 0,28 \\
\hline Matayba elaeagnoides Radlk. Radlk. & Sapindaceae & SI & $\mathrm{Ce} / \mathrm{FA}$ & 1 & 0,08 & 0,44 & 0,31 & 0,28 \\
\hline Bougainvillea glabra Choisy & Nyctaginaceae & $\mathrm{Pi}$ & $\mathrm{Ca} / \mathrm{FA}$ & 1 & 0,08 & 0,4 & 0,31 & 0,26 \\
\hline Miconia cinnamomifolia (DC.) Naudin & Melastomataceae & $\mathrm{Pi}$ & Excl & 2 & 0,15 & 0,29 & 0,31 & 0,25 \\
\hline Indeterminada sp2 & Indeterminada & $\mathrm{NC}$ & $\mathrm{NC}$ & 1 & 0,08 & 0,37 & 0,31 & 0,25 \\
\hline Vitex sellowiana Cham. & Lamiaceae & $\mathrm{Pi}$ & Amp & 3 & 0,23 & 0,2 & 0,31 & 0,25 \\
\hline Vochysia sp1 & Vochysiaceae & $\mathrm{NC}$ & $\mathrm{NC}$ & 1 & 0,08 & 0,24 & 0,31 & 0,21 \\
\hline Myrciaria cuspidata O.Berg & Myrtaceae & SI & Amp & 1 & 0,08 & 0,22 & 0,31 & 0,21 \\
\hline
\end{tabular}


Tabela 1 - Conclusão...

Table 1 - Conclusion...

\begin{tabular}{|c|c|c|c|c|c|c|c|c|}
\hline Espécie & Família & GS & DG & DA & DR & DoR & FR & VI (\%) \\
\hline Eugenia sp1 & Myrtaceae & $\mathrm{NC}$ & $\mathrm{NC}$ & 1 & 0,08 & 0,19 & 0,31 & 0,19 \\
\hline Ixora brevifolia Benth. & Rubiaceae & SI & S/FES & 1 & 0,08 & 0,18 & 0,31 & 0,19 \\
\hline Eugenia uniflora L. & Myrtaceae & $\mathrm{Pi}$ & Amp & 2 & 0,15 & 0,09 & 0,31 & 0,19 \\
\hline Jacaranda brasiliana (Lam.) Pers. & Bignoniaceae & SI & Amp & 1 & 0,08 & 0,16 & 0,31 & 0,19 \\
\hline Syagrus romanzoffiana (Cham.) Glassman & Araceae & $\mathrm{Pi}$ & Amp & 1 & 0,08 & 0,16 & 0,31 & 0,19 \\
\hline $\begin{array}{l}\text { Vernonanthura divaricata (Spreng.) } \\
\text { H.Rob. }\end{array}$ & Asteraceae & Pi & $\mathrm{Ce} / \mathrm{FA}$ & 1 & 0,08 & 0,12 & 0,31 & 0,17 \\
\hline Croton salutaris Casar. & Euphorbiaceae & $\mathrm{Pi}$ & Excl & 2 & 0,15 & 0,03 & 0,31 & 0,17 \\
\hline Platypodium elegans Benth. & Fabaceae & $\mathrm{Pi}$ & Amp & 1 & 0,08 & 0,09 & 0,31 & 0,16 \\
\hline Ocotea corymbosa (Meisn.) Mez & Lauraceae & $\mathrm{Pi}$ & $\mathrm{Ce} / \mathrm{FA}$ & 1 & 0,08 & 0,08 & 0,31 & 0,16 \\
\hline Caesalpinia sp1 & Fabaceae & $\mathrm{NC}$ & $\mathrm{NC}$ & 1 & 0,08 & 0,04 & 0,31 & 0,15 \\
\hline Tovomitopsis sp1 & Clusiaceae & $\mathrm{NC}$ & $\mathrm{NC}$ & 1 & 0,08 & 0,04 & 0,31 & 0,14 \\
\hline Ocotea brachybotrya (Meisn.) Mez & Lauraceae & SI & Excl & 1 & 0,08 & 0,04 & 0,31 & 0,14 \\
\hline $\begin{array}{l}\text { Schefflera morototoni (Aubl.) Maguire et } \\
\text { al. }\end{array}$ & Araliaceae & SI & Amp & 1 & 0,08 & 0,02 & 0,31 & 0,14 \\
\hline Solanum sp1 & Solanaceae & $\mathrm{NC}$ & $\mathrm{NC}$ & 1 & 0,08 & 0,02 & 0,31 & 0,14 \\
\hline Ceiba speciosa (A.St.-Hil.) Ravenna & Malvaceae & $\mathrm{Pi}$ & Amp & 1 & 0,08 & 0,02 & 0,31 & 0,14 \\
\hline Solanum leucodendron Sendtn. & Solanaceae & Pi & Excl & 1 & 0,08 & 0,01 & 0,31 & 0,14 \\
\hline Solanum swartzianum Roem. \& Schult. & Solanaceae & $\mathrm{Pi}$ & $\mathrm{Ce} / \mathrm{FA}$ & 1 & 0,08 & 0,01 & 0,31 & 0,14 \\
\hline Fabaceae sp1 & Fabaceae & $\mathrm{NC}$ & $\mathrm{NC}$ & 1 & 0,08 & 0,01 & 0,31 & 0,14 \\
\hline Persea americana Mill. * & Lauraceae & $\mathrm{NC}$ & Exot & 1 & 0,08 & 0,01 & 0,31 & 0,13 \\
\hline Annona sp1 & Annonaceae & $\mathrm{NC}$ & $\mathrm{NC}$ & 1 & 0,08 & 0,01 & 0,31 & 0,13 \\
\hline Alchornea triplinervia (Spreng.) Müll.Arg. & Euphorbiaceae & $\mathrm{Pi}$ & Amp & 1 & 0,08 & 0,01 & 0,31 & 0,13 \\
\hline Myrtaceae sp3 & Myrtaceae & $\mathrm{NC}$ & $\mathrm{NC}$ & 1 & 0,08 & 0,01 & 0,31 & 0,13 \\
\hline Prunus myrtifolia (L.) Urb. & Rosaceae & SI & Amp & 1 & 0,08 & 0,01 & 0,31 & 0,13 \\
\hline
\end{tabular}

Em que: GS = grupo sucessional (Pi = Pioneira; SI = Secundária Inicial; ST = Secundária Tardia; NC = Não Classificada), DG = distribuição geográfica $($ Excl = exclusiva da Floresta Atlântica; Ce/FA = ocorrentes no Cerrado e Floresta Atlântica; Ca/FA= ocorrentes na Caatinga e Floresta Atlântica; Amp = ocorrentes em três ou mais domínios fitogeográficos (ampla distribuição); S/ FES = nativas do Brasil, porém, não possui distribuição geográfica natural na floresta estacional semidecidual e Exot = exóticas e exóticas invasoras de fora do Brasil), DA = densidade absoluta; $\mathrm{AB}=$ área basal $\left(\mathrm{m}^{2}\right) ; \mathrm{DR}=$ densidade relativa; DoR = dominância relativa; $\mathrm{FR}=$ frequência relativa; VI\% = valor de importância em porcentagem; * espécies exóticas de fora do Brasil.

As famílias mais representativas foram Myrtaceae (14), Fabaceae (13), Rubiaceae (5), Lauraceae (4) e Bignoniaceae (4), juntas somando $47,61 \%$ da riqueza de espécies. Com relação aos gêneros, os mais representativos foram Eugenia (4), Myrcia (4), Ocotea (3), Solanum (3) e Piper (3). As espécies que mais se destacaram quanto ao Valor de Importância (VI) foram, Coffea arabica (14, 93\% do VI total), Nectandra nitidula (9, 74\%), Myrciaria glazioviana (7, 72\%), Cupania oblongifolia (7, 49\%), Erythroxylum citrifolium $(6,81)$, Pseudopiptadenia contorta (4, 86\%) e Artocarpus heterophyllus (4, 10\%), que juntas somam 55, $65 \%$ do total de VI. Além disso, foram encontradas cinco espécies que não são originárias do Brasil: Coffea arabica, Artocarpus heterophyllus, Camellia sinensis, Malvaviscus arboreus e Persea americana, que juntas representam 27,6\% do total de indivíduos da comunidade regenerante (20,5\% do VI Total).

Mesmo se tratando de um pequeno fragmento florestal, as famílias mais representativas encontradas (Myrtaceae, Fabaceae, Rubiaceae, Lauraceae e Bignoniaceae), estão de acordo com os encontrados para trabalhos fitossociológicos de regeneração natural da região (FONSECA; RIBEIRO; 
CARVALHO, 2013; SANTIAGO; FONSECA; CARVALHO, 2014) e com o encontrado por OliveiraFilho, Jarenkow e Rodal (2006), que apontou estas famílias como sendo uma das mais ricas nos domínios da floresta estacional neotropical. Nesse mesmo estudo, Oliveira-Filho, Jarenkow e Rodal (2006), assim como no presente trabalho, também apontaram os gêneros Eugenia, Myrcia, Ocotea e Solanum entre os oito mais ricos no domínio da Floresta Atlântica do Sudeste.

Com relação aos dados da estrutura fitossociológica, os resultados mostram uma comunidade com VI concentrado principalmente nas sete primeiras espécies. Dentre estas, por serem espécies exóticas invasoras (INTER-AMERICAN BIODIVERSITY INFORMATION NETWORK, 2014), duas merecem destaque. A espécie Coffea arabica, com o maior VI, foi a espécie que apresentou o maior número de indivíduos e maior dominância relativa na área. Esta espécie é comum no subbosque de formações florestais e originária das florestas tropicais da Etiópia (KUFA; BURKHARDT, 2010). Apesar de várias décadas após a diminuição das áreas de lavoura de café na região, esta espécie tem sido encontrada com frequência nas florestas do Sudeste do Brasil (LOPES et al., 2002; MARTINS et al., 2008; SANTIAGO; FONSECA; CARVALHO, 2014; RAYMUNDO et al., 2018). No entanto, a sua predominância em formações florestais, em termos de controle e conservação da biodiversidade, é preocupante, uma vez que esta é considerada uma espécie exótica invasora, além de suas plântulas competirem com espécies nativas nos estratos inferiores da formação florestal, o que pode interferir no processo de regeneração (MARTINS et al., 2008).

Já a espécie Artocarpus heterophyllus (jaca), originária das florestas tropicais da região da Índia, já foi registrada tanto em áreas antropizadas quanto em várias unidades de conservação da Floresta Atlântica (FABRICANTE et al., 2012; INTER-AMERICAN BIODIVERSITY INFORMATION NETWORK, 2014). Contudo, o Parque Nacional da Tijuca merece uma especial atenção, pois neste local a agressividade de invasão da jaqueira é bem conhecida, onde já caracteriza um processo de bioinvasão (ABREU; RODRIGUES, 2010). Além disso, Fabricante et al. (2012) alerta para o grau de ameaça à vegetação nativa das áreas invadidas, uma vez que Artocarpus heterophyllus pode alterar a riqueza, diversidade e o solo destas áreas.

$\mathrm{O}$ índice de diversidade de Shannon foi de $\mathrm{H}^{\prime}=3,10$ nats.ind-1, e o de equabilidade de Pielou foi de $J=0,70$. O valor do índice de Shannon ( $H^{\prime}$ ) ficou próximo aos valores obtidos em outros estudos de floresta estacional semidecidual em área urbana e que possuem um tempo menor de regeneração (SALLES; SCHIAVINI, 2007; FONSECA; RIBEIRO; CARVALHO, 2013; SANTIAGO; FONSECA; CARVALHO, 2014). Complementarmente, o índice de equabilidade (Pielou) encontrado $\left(\mathrm{J}^{\prime}=0,70\right)$ revela que apenas $70 \%$ da diversidade $\left(\mathrm{H}^{\prime}\right)$ hipotética máxima foi atingida, ou seja, indica uma disparidade na proporção dos indivíduos entre as populações, nas quais algumas espécies, devido à baixa densidade das demais, concentram um grande número de seus indivíduos. Este fato fica evidente quando somado à densidade relativa das cinco primeiras espécies com o maior VI, que juntas concentram uma DR superior a $50 \%$.

Com relação às análises multivariadas, a análise de agrupamento revelou a formação de alguns grupos florísticos bem definidos (Figura 2), com destaque principal para um grupo de dez parcelas (P2, P3, P4, P5, P6, P7, P8, P17, P24 e P25), nas quais as espécies de maior peso para formação deste grupo foram Coffea arabica, Artocarpus heterophyllus, Myrciaria glazioviana e Cupania oblongifolia. Percebe-se que a comunidade apresenta uma relativa heterogeneidade interna, levando em consideração o limite de divisão significativo como $>0,5$, utilizado para florestas heterogêneas (FELFILI et al., 2011). De forma complementar, a análise de correspondência distendida (DCA) indicou a presença de gradientes fortes na comunidade (Eixo 1 =0,56; Eixo 2=0,31) (Figura 3). Considerando o conceito de autovalor significativo como > 0,3 (FELFILI et al., 2011), ficou evidente a formação de gradientes na comunidade. Apesar do tamanho reduzido do fragmento florestal estudado, as espécies estão distribuídas em sítios preferenciais, isto é, há uma ocupação diferenciada de nichos na comunidade (FELFILI et al., 2011), evidenciado na análise pela formação de alguns grupos de espécies e parcelas. 
Figura 2 - Análise de agrupamento de espécies entre as parcelas pelo coeficiente de Morisita-Horn (quantitativo); correlação cofenética $=\mathbf{0 , 7 8}$. Método de agrupamento $=$ UPGMA.

Figure 2 - Species cluster analysis between plots by Morisita-Horn coefficient (quantitative); cophenetic correlation $=0.78$. Clustering method $=$ UPGMA.

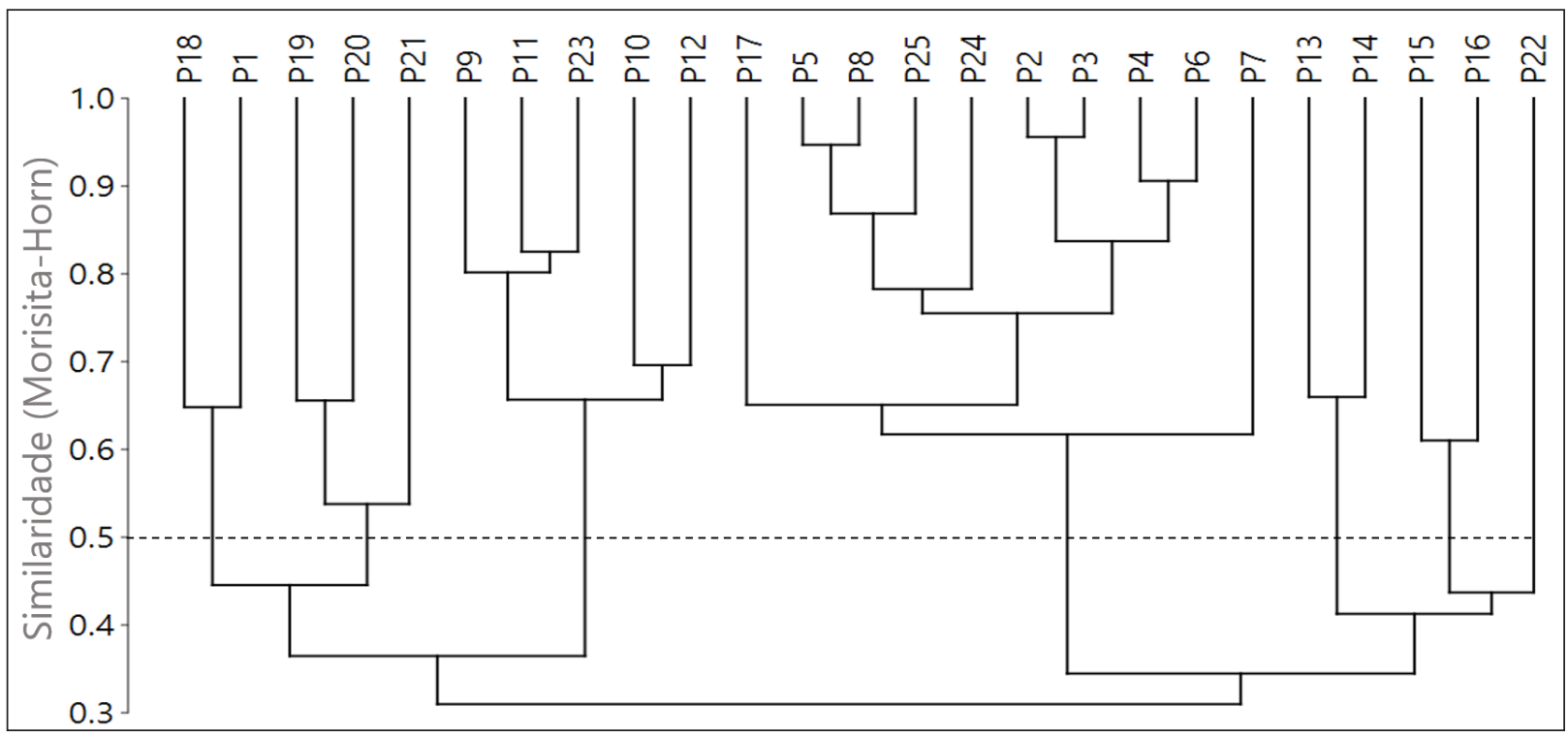

Figura 3 - Diagrama de ordenação das parcelas resultante da Análise da correspondência distendida (DCA). Autovalores: Eixo $1=0,56$; Eixo $2=0,31$. A saber $=$ quadrados simbolizam as unidades amostrais (parcelas) e os círculos as espécies amostradas.

Figure 3 - Diagram of plots ordination from the distended correspondence analysis (DCA). Eigenvalues $=$ Axis $1=0.56$; Axis $2=0.31$.

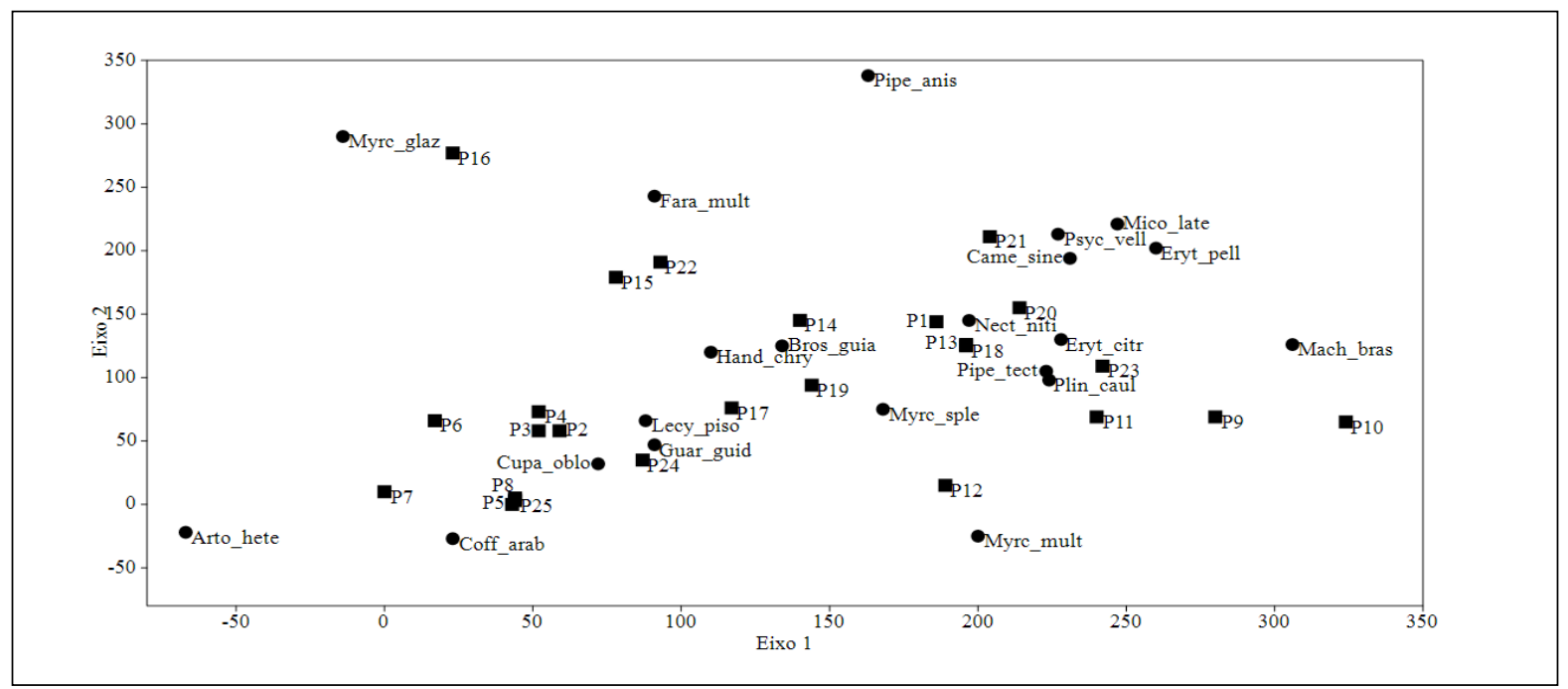

A distribuição geográfica das espécies identificadas mostrou uma predominância de espécies de ampla ocorrência, tanto na abundância $(47,1 \%)$, quanto na riqueza $(48,6 \%)$, seguida pelas espécies exóticas de fora do Brasil (28,1\% - abundância; 7,1\% - riqueza), concomitantemente pelas nativas do Brasil, porém, que não ocorrem naturalmente na floresta estacional semidecidual $(15,7 \%$ - abundância; $10,0 \%$ - riqueza). Além disso, foram encontradas 13 espécies que ocorrem no domínio do Cerrado e Floresta Atlântica (5,8\%), 10 espécies exclusivas da Floresta Atlântica (3,3\%) e apenas uma que se distribui tanto na Caatinga quanto na Floresta Atlântica $(0,1 \%$ ) (Figura 4-A). O resultado aponta para uma flora com 
maior tolerância a ambientes distintos e até mesmo às perturbações antrópicas em ambiente urbano. Com relação às espécies nativas do Brasil, entretanto, que não ocorrem na fitofisionomia do presente estudo, justifica-se pelo fato do local ser um Parque, passível de eventual manejo antrópico. A inserção de espécies exóticas em projetos paisagísticos é prática comum, e a grande maioria dos planejadores preferem o uso de espécies exóticas, tanto de outras regiões do país como estrangeiras para incorporar em seus projetos, ignorando a grande variedade de espécies nativas de cada região (GONÇALVES; PAIVA, 2004).

A análise dos grupos sucessionais apontou uma comunidade com predominância de grupos sucessionais iniciais, isto é, secundárias iniciais com 36,7\% do total de indivíduos e 33,3\% do total de espécies, seguido das pioneiras com $30,6 \%$ dos indivíduos e 36,9\% das espécies. Vale ressaltar também, o grande número de indivíduos no grupo das exóticas, que representam um total de 27,6\% (Figura 4B). O grande número de espécies e indivíduos pertencentes a grupos sucessionais iniciais, aponta para uma comunidade em estágio inicial de sucessão. As espécies pioneiras e secundárias iniciais são espécies que dependem de certa quantidade de luz para realizarem seus processos fisiológicos (CHAZDON, 2008; OLIVEIRA- FILHO; SCOLFORO, 2008), desta forma, como a área de estudo não apresenta um dossel fechado e possui um histórico de perturbação antrópico, permite que a energia luminosa atinja o solo em quantidades suficientes para favorecer esse grupo de espécies. Corroborando estes dados, Chazdon (2008) comenta que a presença elevada de espécies pertencentes a grupos sucessionais iniciais é uma característica peculiar das florestas antropicamente perturbadas.

Figura 4 - Abundância (\%) e riqueza de espécies (\%) regenerantes amostradas no fragmento Florestal do Parque Mariano Procópio, Juiz de Fora - Minas Gerias, de acordo com sua (A) Distribuição geográfica e (B) Grupos Sucessionais. Siglas de acordo com a Tabela 1.

Figure 4 -Abundance (\%) and species richness (\%) sampled in the regeneration of the forest fragment of Park 'Mariano Procópio', Juiz de Fora - Minas Gerais state, according to your (A) Geographic distribution and (B) Successional groups. Acronyms according to Table 1.

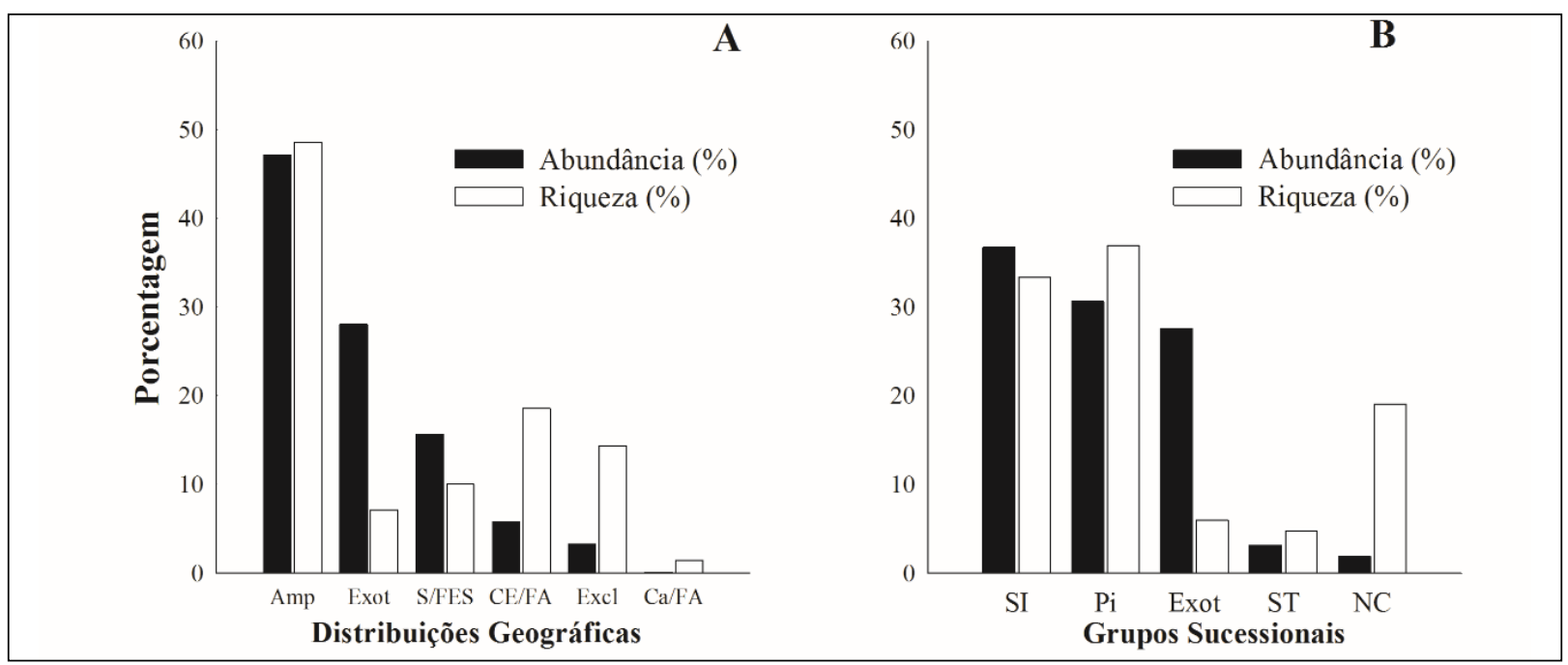

A trajetória sucessional de uma comunidade florestal é influenciada por diversos fatores (CHAZDON, 2003), dentre estes merecem destaque, a frequência e intensidade dos distúrbios ou usos da terra anteriores, natureza da vegetação remanescente e condições pós-distúrbio, como por exemplo, tipos de manejo, colonização por espécies exóticas invasoras, além da possibilidade de dispersão de sementes a partir da matriz florestal do entorno (CHAZDON, 2003; 2008). Diante do exposto, tais resultados apontam que, mesmo após aproximadamente 150 anos de regeneração, a floresta ainda se encontra em estágio inicial de sucessão. O impacto antrópico constante e a presença significativa de espécies exóticas provavelmente têm prejudicado o desenvolvimento da floresta e impedindo seu avanço para estágios sucessionais mais tardios. Desta forma, partindo do encontrado neste trabalho e sabendo dos riscos acarretados pelas espécies exóticas invasoras, os resultados podem 
auxiliar gestores na tomada de decisões relativas às medidas preventivas de controle ou erradicação das exóticas, propiciando que a floresta avance no processo sucessional.

\section{Conclusões}

A comunidade regenerante apresentou uma flora típica das florestas estacionais semideciduais, contudo, entre os maiores Valores de Importância destacam-se duas espécies exóticas invasoras (Coffea arabica e Artocarpus heterophyllus). O valor da diversidade de Shannon ficou próximo a outros estudos em fragmentos florestais com tempo menor de regeneração e as análises multivariadas indicaram uma comunidade com relativa heterogeneidade interna e com gradientes ambientais fortes. Já a distribuição geográfica das espécies apontou para uma flora com distribuição em três ou mais domínios fitogeográficos, tanto na abundância quanto na riqueza. Os grupos sucessionais indicaram uma predominância de indivíduos e espécies no grupo das secundárias iniciais e pioneiras. As informações levantadas mostram a relevância do fragmento florestal, tanto quanto a expressiva diversidade florística, quanto com sua representatividade ecológica. Todavia, mesmo com 150 anos de regeneração, o fragmento florestal do Parque Mariano Procópio aparenta estar com dificuldades em avançar para estágios sucessionais superiores. Tal fato é corroborado pela expressiva presença de indivíduos pertencentes a grupos sucessionais iniciais, e, principalmente, pela forte presença de espécies exóticas reconhecidamente invasoras na Floresta Atlântica. Além disso, este estudo é importante para reforçar o risco da implantação de espécies exóticas invasoras em projetos paisagísticos.

\section{Agradecimentos}

Agradecemos aos colegas do Herbário CESJ e do Laboratório de Ecologia Vegetal (Departamento de Botânica/UFJF) pela ajuda na identificação e nos trabalhos de campo; aos taxonomistas Daniele Monteiro e Pedro Luís Rodrigues de Moraes pela identificação das Piperaceae e Lauraceae, respectivamente; à Fundação Mariano Procópio (MAPRO) pela logística e licença de coleta no Parque; à CAPES, ao CNPq (Bolsa de Produtividade em Pesquisa a F.A. Carvalho) e a FAPEMIG pelo apoio financeiro.

\section{Referências}

ABREU, R. C. R.; RODRIGUES, P. J. F. P. Exotic tree Artocarpus heterophyllus (Moraceae) invades the Brazilian Atlantic Rainforest. Rodriguésia, Rio de Janeiro, v. 61, n. 4, p. 677-688, 2010.

ALVEY, A. A. Promoting and preserving biodiversity in the urban forest. Urban Forestry \& Urban Greening, Amsterdam, v. 5, n. 4, p. 195-201, 2006.

ANGIOSPERM PHYLOGENY GROUP. An update of the Angiosperm Phylogeny Group classification for the orders and families of flowering plants: APG IV. Botanical Journal of the Linnean Society, London, v. 181, n. 1, p. 1-20, 2016.

BANDEIRA, C. M. Parque Nacional da Tijuca. São Paulo: Markom Books, 1993. 170 p.

CHAZDON, R. L. Tropical Forest recovery: legacies of human impact and disturbances. Perpectives in Plant Ecology, Evolution and Systematics, Jena, v. 6, n. 1-2, p. 51-71, 2003.

CHAZDON, R. L. Chance and determinism in tropical forest succession. In: CARSON, W. P.; SCHNITZER, S. A. (Ed.). Tropical forest community ecology. Chichester: Blackwell, 2008. 536 p.

DELPHIM, C. F. M. O Parque Mariano Procópio. In: DELPHIM, C. F. M. Leituras Paisagísticas: teoria e práxis/ Glaziou. Rio de Janeiro: Ed. UFRJ, 2007. 238 p.

DRUMMOND, G. M. et al. Biodiversidade em Minas Gerais: um atlas para sua conservação. 2. ed. Belo Horizonte: Fundação Biodiversitas, 2005. 222 p. 
DURIGAN, G.; ENGEL, V. L. Restauração de Ecossistemas no Brasil: onde estamos e para onde podemos ir? In: MARTINS, S. V. Restauração ecológica de ecossistemas degradados. Viçosa, MG: Editora UFV, 2012. 293 p.

FABRICANTE, J. R. et al. Invasão biológica de Artocarpus heterophyllus Lam. (Moraceae) em um fragmento de Mata Atlântica no Nordeste do Brasil: impactos sobre a fitodiversidade e os solos dos sítios invadidos. Acta Botanica Brasilica, Feira de Santana, v. 26, n. 2, p. 399-407, 2012.

FELFILI, J. M. et al. Análise multivariada: princípios e métodos em estudos de vegetação. In: FELFILI, J. M.; EISENLOHR, P. V. et al. (Ed.). Fitossociologia no Brasil: métodos e estudos de casos. Viçosa: Ed. UFV, 2011. 556 p.

FONSECA, S. N.; RIBEIRO, J. H. C.; CARVALHO, F. A. Estrutura e diversidade da regeneração arbórea em uma Floresta Secundária Urbana (Juiz de Fora, MG, Brasil). Floresta e Ambiente, Seropédica, v. 20, n. 3, p. 307-315, 2013.

GONÇALVES, W.; PAIVA, H. N. Árvores para ambiente urbano. Viçosa, MG: Aprenda Fácil, 2004. $242 \mathrm{p}$

HAMMER, O. et al. paleontological Statistics software package for education and data analysis. Palaeontologia Electronica, Oslo, v. 4, n. 1, p. 1-9, 2001.

IBGE. Manual técnico da vegetação brasileira. 2. ed. Rio de Janeiro: Instituto Brasileiro de Geografia e Estatística, 2012. 271 p.

INTER-AMERICAN BIODIVERSITY INFORMATION NETWORK. Base de dados nacional de espécies exóticas invasoras. Florianópolis: Instituto Hórus de Desenvolvimento e Conservação Ambiental, 2014. Disponível em: http://i3n.institutohorus.org.br. Acesso em: 3 jun. 2016.

JUIZ DE FORA (MG). Companhia De Saneamento Municipal. Hidrografia em Juiz de Fora. Disponível em: http://www.cesama.com.br/?pagina=hidrografia. Acesso em: 16 jun. 2016.

KENT, M.; COKER, P. Vegetation description and data analysis: a practical approach. London: John Wiley \& Sons, 1992. 365 p.

KUFA, T.; BURKHARDT, J. Hydraulic characteristics of wild Coffea arabica populations in natural forests of Ethiopia. Gene Conserve, Brasília, v. 9, n. 37, p. 124-135, 2010.

LOPES, W. P. et al. Composição da flora arbórea de um trecho de floresta estacional no Jardim Botânico da Universidade Federal de Viçosa (face sudoeste), Viçosa, Minas Gerais. Revista Árvore, Viçosa, MG, v. 26, n. 3 , p. 339-347, 2002.

MAGURRAN, A. E. Measuring biological diversity. Oxford: Blackwell, 2004.

MARTINS, S. V. et al. Caracterização do dossel e do estrato de regeneração natural no sub-bosque e em clareiras de uma florestal estacional semidecidual no município de Viçosa, MG. Revista Árvore, Viçosa, MG, v. 32, n. 4, p.759-767, 2008.

MCKINNEY, M. Urbanization as a major cause of biotic homogenization. Biological Conservation, Boston, v. 127, n. 3, p. 247-260, 2006.

MEIRA-NETO, J. A. A. et al. Estrutura de uma Floresta Estacional Semidecidual insular em área diretamente afetada pela usina hidrelétrica de Pilar, Ponte Nova, Zona da Mata de Minas Gerais. Revista Árvore, Viçosa, MG, v. 21, n. 4, p. 493-500, 1997.

MELI, P. et al. Optimizing seeding density of fast-growing native trees for restoring the Brazilian Atlantic Forest. Restoration Ecology, Washington, v. 26, n. 2, p. 212-219, 2017.

OLIVEIRA-FILHO, A. T.; JARENKOW. J. A.; RODAL, M. J. N. Floristic relationships of seasonally dry forests of eastern South America based on tree species distribution patterns. In: PENNINGTON, R. T.; RATTER, J. A.; LEWIS, G. P. (Ed.). Neotropical savannas and dry forests: plant diversity, biogeography and conservation. Boca Raton: CRC Press, 2006. 491 p. 
OLIVEIRA-FILHO, A. T.; SCOLFORO, J. R. Inventário florestal de Minas Gerais: espécies arbóreas da flora nativa. Lavras: Ed. UFLA, 2008. 418 p.

PIMENTEL, D. et al. Economic and environmental threats of alien plant, animal, and microbe invasions. Agriculture, Ecosystems \& Environment, Amsterdam, v. 84, n. 1, p. 1-20, 2001.

PERRING, M. P. et al. Novel urban ecosystems and ecosystem services. In: HOBBS, R. J.; HIGGS, E. S.; HALL, C. M. (Ed.). Novel ecosystems: intervening in the new ecological world order. Chichester: John Wiley \& Sons, 2013. 380 p.

RAYMUNDO, D. et al. Persistence of Coffea arabica and its relationship with the structure, species diversity and composition of a secondary forest in Brazil. PLoS ONE, São Francisco, v. 13, n. 3, p. 1-15, 2018.

RODRIGUES, R. R. et al. On the restoration of high diversity forests: 30 years of experience in the Brazilian Atlantic Forest. Biological Conservation, Boston, v. 142, n. 6, p. 1242-1251, 2009.

SALLES, J. C.; SCHIAVINI, I. Estrutura e composição do estrato de regeneração em um fragmento florestal urbano: implicações para a dinâmica e a conservação da comunidade arbórea. Acta Botânica Brasílica, Feira de Santana, v. 21, n. 1, p. 223-233, 2007.

SANTIAGO, D. S.; FONSECA, C. R.; CARVALHO. F. A. Fitossociologia da regeneração natural de um fragmento urbano de Floresta Estacional Semidecidual (Juiz de Fora, MG). Revista Brasileira de Ciências Agrárias, Pernambuco, v. 9, n. 1, p. 117-123, 2014.

VALVERDE, O. Estudo Regional da Zona da Mata de Minas Gerais. Revista Brasileira de Geografia, Rio de Janeiro, v. 20, n. 1, p. 1-82, 1958. 